FSU-981218

BNL-HET-98/48

December, 1998

\title{
ASSOCIATED HIGGS PRODUCTION IN THE MSSM
}

\author{
S. Dawson \\ Physics Department, Brookhaven National Laboratory, \\ Upton, NY 11973, USA \\ L. Reina \\ Physics Department, Florida State University, \\ Tallahassee, FL 32306, USA
}

\begin{abstract}
We investigate the inclusive production of the Higgs bosons of the minimal supersymmetric model with a heavy quark pair, $(t \bar{t}$ and $b \bar{b})$, in $e^{+} e^{-}$collisions at high energies, $\sqrt{s}=500 \mathrm{GeV}$ and $\sqrt{s}=1 \mathrm{TeV}$. The $\mathcal{O}\left(\alpha_{s}\right)$ QCD radiative corrections are included and the dependence of the production rates on the parameters of the MSSM is explored. The associated production of a Higgs boson with a $b \bar{b}$ pair receives large resonant contributions and can have a significant rate.
\end{abstract}




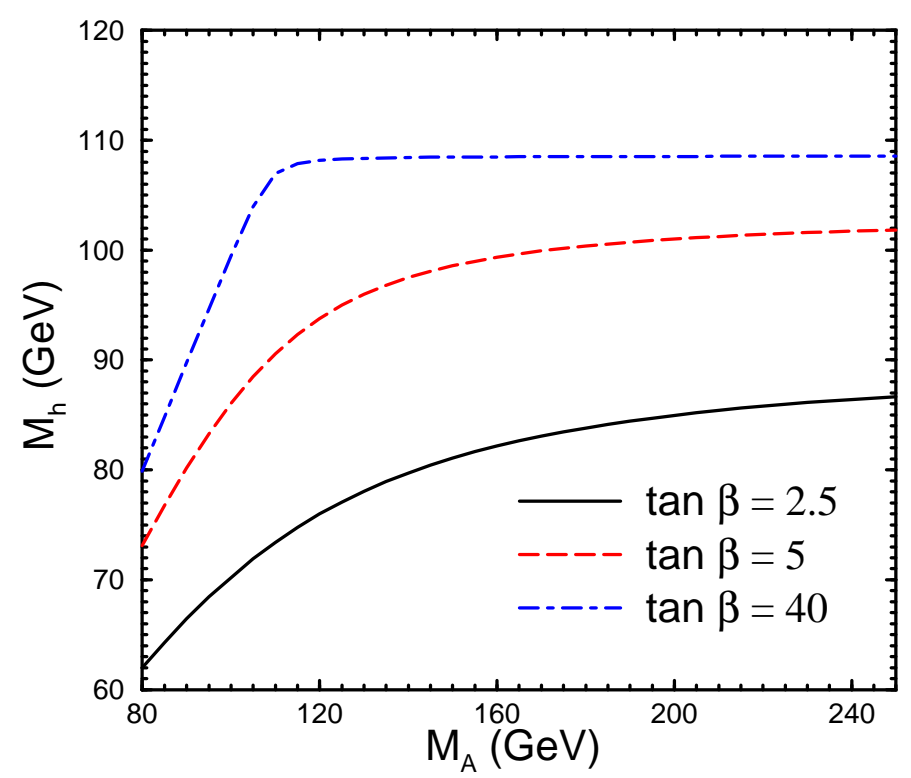

Figure 1: Mass of the lightest neutral Higgs boson as a function of the pseudoscalar mass $M_{A}$. The squarks are assumed to have a common mass, $M_{S}=500 \mathrm{GeV}$ and the scalar mixing parameters are set to zero.

\section{Introduction}

The search for the Higgs boson is an important objective of present and future colliders. In the simplest version of the Standard Model, the Higgs boson is necessary in order to understand fermion and gauge boson masses. Once the Higgs boson has been discovered, it is crucial to measure its couplings to fermions and gauge bosons. The couplings to the gauge bosons can be measured through the associated production processes, $e^{+} e^{-} \rightarrow Z \phi, q \bar{q}^{\prime} \rightarrow W^{ \pm} \phi$, and $q \bar{q} \rightarrow Z \phi$, and through vector boson fusion, $W^{+} W^{-} \rightarrow \phi$ and $Z Z \rightarrow \phi$, (where $\phi$ is a generic Higgs boson). The couplings of the Higgs boson to fermions are more difficult to measure, however.

In the Standard Model, the fermion-Higgs couplings are completely determined in terms of the fermion masses,

$$
g_{Q Q h_{S M}}=-\frac{M_{Q}}{v},
$$

where $v=\left(\sqrt{2} G_{F}\right)^{-1 / 2}$ and so the top quark-Higgs boson Yukawa coupling is large while the bottom quark-Higgs boson Yukawa coupling is much smaller. In extensions of the Standard Model, however, the Yukawa couplings can be significantly different. They are no longer strictly 
proportional to the fermion masses, but depend on the parameters of the model. The minimal supersymmetric model (MSSM) provides a useful benchmark for comparison of the $Q \bar{Q} \phi$ couplings with those of the Standard Model.

In fact, the process $e^{+} e^{-} \rightarrow t \bar{t} \phi$ provides a direct mechanism for measuring the top quarkHiggs Yukawa coupling, and for discriminating the Standar Model type Yukawa coupling from others. [1,2] This process proceeds via $\gamma$ and $Z$ exchange, with the Higgs boson bremsstrahlung from the heavy quark. There is also a contribution from the Higgs boson coupling to the exchanged $Z$, but this is always less than a few percent and so does not upset the interpretation of this process as a measurement of the top quark-Higgs Yukawa couplings.

The dominant production mechanism for a Higgs boson at a hadron machine is gluon fusion, $g g \rightarrow \phi$, which proceeds by a loop diagram containing heavy fermions and so is also sensitive to the fermion-Higgs Yukawa couplings. For heavy fermions, however, the gluon fusion process is independent of the fermion mass and so this mechanism counts the number of heavy fermions which couple to the Higgs boson. The process $g g \rightarrow \phi$ thus does not provide an unambiguous determination of the coupling of the Higgs boson to heavy fermions since it is sensitive to the unknown number of heavy fermions. In addition there are uncertainties due to the fact that QCD corrections to the $g g \rightarrow h$ process are large.

In a previous paper [3], we computed the $\mathcal{O}\left(\alpha_{s}\right)$ corrections to the process $e^{+} e^{-} \rightarrow t \bar{t} h_{S M}$. At $\sqrt{s}=500 \mathrm{GeV}$, they are large and positive, while at $\sqrt{s}=1 \mathrm{TeV}$, the QCD corrections are small. In this paper, we apply the $\mathcal{O}\left(\alpha_{s}\right)$ corrections [3, [4 to the production of the Higgs bosons of the MSSM and explore the dependence of the production rates on the parameters of the MSSM.

It is more difficult to extract the bottom quark-Higgs Yukawa coupling from a measurement of $e^{+} e^{-} \rightarrow b \bar{b} h_{S M}$, since the coupling itself is tiny and the $Z$ contribution is relevant. However in the MSSM, for certain values of $\tan \beta$, the ratio of the neutral Higgs vacuum expectation values, the $b \bar{b} \phi$ Yukawa coupling receives significant enhancements and so the process $e^{+} e^{-} \rightarrow b \bar{b} \phi$ may be larger than in the Standard Model. Moreover, there are 5 Higgs bosons in the MSSM, so that additional processes not present in the Standard Model may be useful to pin down the fermion-Higgs boson Yukawa couplings. In particular, there are new resonance contributions, for example $e^{+} e^{-} \rightarrow A^{0} h_{i}, A^{0} \rightarrow b \bar{b}$, which are not present in the Standard Model. The effects of these new contributions can be significant and are the focus of this paper.

The process $e^{+} e^{-} \rightarrow b \bar{b} \phi$ is not only sensitive to the b-quark Higgs Yukawa coupling but also has an interesting dependence on the other parameters of the supersymmetric model. Sections 5 and 6 explore this dependence in detail.

In Section 2, we provide an overview of the elements of the MSSM relevant for our study. The following sections describe our results for the associated production of the scalars and pseudoscalar of the MSSM with $t \bar{t}$ and $b \bar{b}$ pairs. Section $\overline{7}$ contains some conclusions. 


\section{SUSY Background}

The minimal supersymmetric version of the standard model (MSSM) is a theoretically appealing extension of the Standard Model and provides a useful framework for comparison with the Standard Model. In the MSSM, the Higgs sector contains two $S U(2)_{L}$ doublets and so there are five physical Higgs bosons; two neutral scalars, $h^{0}$ and $H^{0}$, (which we will often denote by $h_{i}^{0}$ ), a pseudo-scalar $A^{0}$, and a pair of charged scalars, $H^{ \pm}$. [5] We are therefore led to consider the processes,

$$
\begin{aligned}
& e^{+} e^{-} \rightarrow Q \bar{Q} h^{0}, \\
& e^{+} e^{-} \rightarrow Q \bar{Q} H^{0} \\
& e^{+} e^{-} \rightarrow Q \bar{Q} A^{0} \\
& e^{+} e^{-} \rightarrow \bar{t} b H^{+}, t \bar{b} H^{-}
\end{aligned}
$$

where $Q$ is either $t$ or $b$. These processes have been previously considered in Ref. [2] and we extend their analysis. The rates are small, but the signatures are distinctive.

At lowest order, all masses and couplings in the Higgs sector can be computed in terms of two parameters, which are typically taken to be $M_{A}$, the mass of the pseudoscalar Higgs boson, and $\tan \beta$, the ratio of vacuum expectation values. At higher order, there is a dependence on the scalar masses and mixing parameters. Having picked $M_{A}$, $\tan \beta$, and the scalar masses and mixing parameters, the neutral Higgs boson masses are predicted [ [6] 8 ].

The MSSM has the feature that there is an upper limit on the mass of the lightest neutral Higgs boson. Including the radiative corrections to leading logarithm, we have,

$$
M_{h}^{2}<M_{Z}^{2} \cos ^{2} 2 \beta+\delta
$$

with

$$
\delta=\frac{3 G_{F} M_{t}^{4}}{\sqrt{2} \pi^{2}} \log \left(1+\frac{M_{S}^{2}}{M_{t}^{2}}\right),
$$

and $M_{S}$ is a common squark mass. In our numerical studies the renormalization group improved values for the Higgs boson masses, along with the NLO corrections are included, using the program HDECAY. 9] Fig. 1 shows the mass of the lightest neutral Higgs boson as a function of $M_{A}$. There is clearly a maximum value around $M_{h}=110 \mathrm{GeV}$. Including mixing effects in the squark and Higgs sectors raises this limit to around $130 \mathrm{GeV}$. Such a light Higgs boson

\footnotetext{
${ }^{1}$ The processes $e^{+} e^{-} \rightarrow \bar{t} b H^{+}, t \bar{b} H^{-}$are interesting in the small mass region where $H^{ \pm}$cannot decay to $t$ nor $t$ decay to $H^{ \pm}$[2]. Outside this mass region, other processes are more useful for determining the Yukawa couplings. [2] We will not consider these processes further.
} 
Table 1: Higgs boson couplings to fermions in units of $g_{Q Q h_{S M}} \equiv-M_{Q} / v$

\begin{tabular}{|lccc|}
\hline$Q$ & $C_{Q Q h}$ & $C_{Q Q H}$ & $C_{Q Q A}$ \\
\hline$t$ & $\frac{\cos \alpha}{\sin \beta}$ & $\frac{\sin \alpha}{\sin \beta}$ & $\cot \beta$ \\
$b$ & $-\frac{\sin \alpha}{\cos \beta}$ & $\frac{\cos \alpha}{\cos \beta}$ & $\tan \beta$ \\
\hline
\end{tabular}

may be observable in association with a heavy quark pair, providing additional impetus to our study.

The LEP2 experiments have searched for the processes $e^{+} e^{-} \rightarrow Z h^{0}$ and $e^{+} e^{-} \rightarrow h^{0} A^{0}$ and find the $95 \%$ confidence level limits, [10]

$$
\begin{aligned}
M_{A} & >78 \mathrm{GeV}, \\
\tan \beta & >2.1,
\end{aligned}
$$

for the case with no mixing in the squark sector.[? We will therefore consider $\tan \beta=2.5,5$ and 40 and $M_{A}>80 \mathrm{GeV}$ as representative values in our study.f The corresponding values for the light Higgs mass, $M_{h}$, can be found from Fig. 国.

An important feature of SUSY models is that the fermion-Higgs couplings are no longer strictly proportional to mass as they are in the Standard Model. It is convenient to write the couplings for the neutral Higgs bosons to the fermions in terms of the Standard Model Higgs boson couplings. In particular, we write the couplings of the Higgs bosons to the quarks as

$$
\mathcal{L}=-\left(\sqrt{2} G_{F}\right)^{1 / 2} M_{i}\left[C_{Q Q h} \bar{Q}_{i} Q_{i} h^{0}+C_{Q Q H} \bar{Q}_{i} Q_{i} H^{0}+C_{Q Q A} \bar{Q}_{i} \gamma_{5} Q_{i} A^{0}\right]
$$

where $C_{Q Q h}$ is 1 for a Standard Model Higgs boson, $\left(C_{Q Q H}=C_{Q Q A}=0\right)$, while the corresponding MSSM couplings are given in Table 1. The fermion-Higgs boson Yukawa couplings in the MSSM are then,

$$
\begin{aligned}
g_{Q Q h_{i}} & =-\left(\sqrt{2} G_{F}\right)^{1 / 2} M_{Q} C_{Q Q h_{i}}, \\
g_{Q Q A} & =-\left(\sqrt{2} G_{F}\right)^{1 / 2} M_{Q} C_{Q Q A} .
\end{aligned}
$$

For small $M_{A}$, the couplings of the neutral Higgs boson to fermions can be significantly different from the Standard Model couplings; the $b$-quark coupling to the lighter of the neutral Higgs

\footnotetext{
${ }^{2}$ There is also a small allowed region with $\tan \beta<0.8$. 110

${ }^{3}$ Results from precision electroweak measurements suggest that the true bound on $M_{A}$ may be slighter than this. 11].

${ }^{4}$ Of course, for a given value of $\tan \beta$, the lower bound on $M_{A}$ may be greater than that of Eq. 5 .
} 


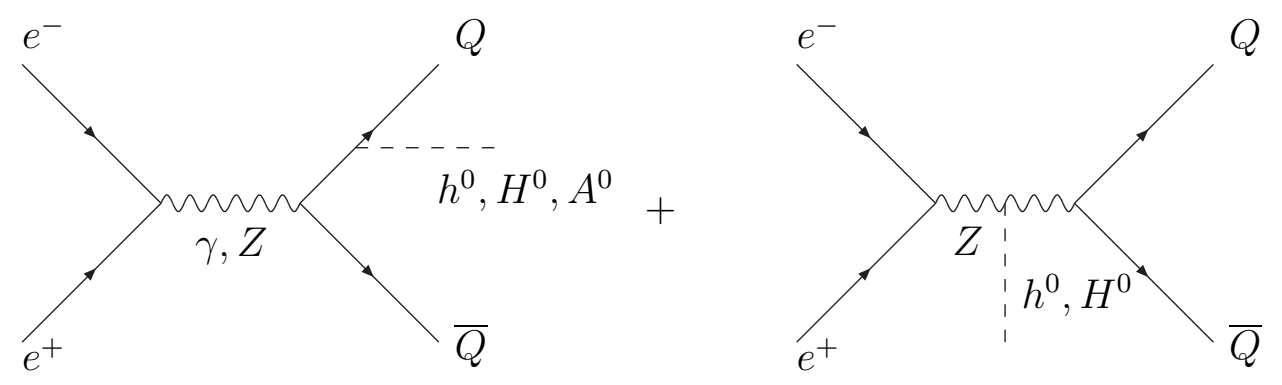

Figure 2: Feynman diagrams contributing to the lowest order processes, $e^{+} e^{-} \rightarrow Q \bar{Q} h_{i}^{0}, A^{0}$ in the MSSM.
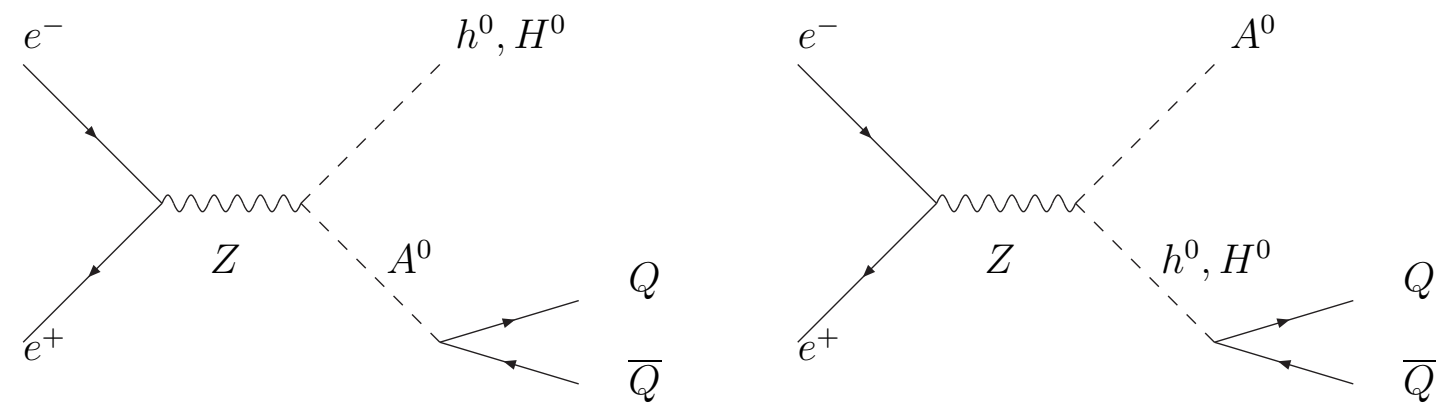

Figure 3: Feynman diagrams contributing to $e^{+} e^{-} \rightarrow Q \bar{Q} h_{i}^{0}, A^{0}$ which are not present in the Standard Model.

bosons, $h^{0}$, becomes enhanced, while the $t$-quark coupling to $h^{0}$ is suppressed for large $\tan \beta$. On the other hand, the coupling of the heavier neutral Higgs boson, $H^{0}$, to the $b$ is enhanced at small $\tan \beta$ and the coupling to $t$ enhanced at large $\tan \beta$ for light $M_{A}$.

The angle $\alpha$ is related to the mixing in the neutral Higgs boson mass matrix. In the leading logarithmic approximation,

$$
\tan 2 \alpha=\frac{\left(M_{A}^{2}+M_{Z}^{2}\right) \sin 2 \beta}{\left(M_{A}^{2}-M_{Z}^{2}\right) \cos 2 \beta+\delta / \sin ^{2} \beta},
$$

where $-\pi / 2<\alpha<0$. In our numerical studies we include the renormalization group improved values for $\alpha$ using the program HDECAY. [9]

\section{$3 \quad e^{+} e^{-} \rightarrow t \bar{t} h_{i}^{0}$}

The rate for $e^{+} e^{-} \rightarrow t \bar{t} h_{i}^{0}, h_{i}^{0}=h^{0}, H^{0}$, receives the contributions from $\gamma$ and $Z$ exchange shown in Fig. 2 as in the Standard Model. In the MSSM, there is an additional contribution not present in the Standard Model from the sub-process $e^{+} e^{-} \rightarrow A^{0} h_{i}^{0}, A^{0} \rightarrow t \bar{t}$, shown in Fig. 3 . 
The rate for the associated production of a neutral SUSY Higgs boson with a heavy quark can be written as 2, 3]

$$
\begin{aligned}
\frac{d \sigma\left(e^{+} e^{-} \rightarrow Q \bar{Q} h_{i}^{0}\right)}{d x_{h}}= & N_{c} \frac{\sigma_{0}}{(4 \pi)^{2}}\left\{\left[Q_{e}^{2} Q_{Q}^{2}+\frac{2 Q_{e} Q_{Q} v_{e} v_{Q}}{1-M_{Z}^{2} / s}+\frac{\left(v_{e}^{2}+a_{e}^{2}\right)\left(v_{Q}^{2}+a_{Q}^{2}\right)}{\left(1-M_{Z}^{2} / s\right)^{2}}\right] G_{1}\right. \\
& +\frac{v_{e}^{2}+a_{e}^{2}}{\left(1-M_{Z}^{2} / s\right)^{2}}\left[a_{Q}^{2} \sum_{i=2}^{6} G_{i}+v_{Q}^{2}\left(G_{4}+G_{6}\right)+\frac{G_{7}}{16 c_{W}^{2} s_{W}^{2}}\right] \\
& \left.+\frac{Q_{e} Q_{Q} v_{e} v_{Q}}{1-M_{Z}^{2} / s} G_{6}\right\},
\end{aligned}
$$

where $\sigma_{0}=4 \pi \alpha^{2} / 3 s, \alpha$ is the QED fine structure constant, $N_{c}=3$ is the number of colors, $x_{h}=2 E_{h} / \sqrt{s}$ with $E_{h}$ the energy of the Higgs boson $h_{i}^{0}$, and $Q_{i}, v_{i}$ and $a_{i}(i=e, Q)$ denote the electromagnetic and weak couplings of the electron and of the heavy quark respectively,

$$
v_{i}=\frac{2 I_{3 L}^{i}-4 Q_{i} s_{W}^{2}}{4 s_{W} c_{W}} \quad, \quad a_{i}=\frac{2 I_{3 L}^{i}}{4 s_{W} c_{W}}
$$

with $I_{3 L}^{i}= \pm 1 / 2$ being the weak isospin of the left-handed fermions and $s_{W}^{2}=1-c_{W}^{2}=0.23$. The contribution from the $Z A^{0} h_{i}^{0}$ coupling shown in Fig. 3 and its interference with the other contributions is given by the factor $G_{7}$. For completeness we list the coefficients $G_{i}\left(x_{h}\right)$ in Appendix A.

In the case of $t \bar{t} h^{0}$ and $t \bar{t} H^{0}$ production, the dominant contribution at $\sqrt{s}=500 \mathrm{GeV}$ is photon exchange, as is the case also in the Standard Model. In Fig. 1 , we show the ratio of the cross section computed including only the photon exchange to the total cross section for the case of $e^{+} e^{-} \rightarrow t \bar{t} h^{0}$. Very similar results hold for the $e^{+} e^{-} \rightarrow t \bar{t} H^{0}$ process. Since the cross section is dominated by photon exchange, the contributions from $e^{+} e^{-} \rightarrow A^{0} h_{i}^{0} \rightarrow t \bar{t} h_{i}^{0}$ and $e^{+} e^{-} \rightarrow Z \rightarrow t \bar{t} h_{i}^{0}$ can safely be neglected at $\sqrt{s}=500 \mathrm{GeV}$.

The $\mathcal{O}\left(\alpha_{s}\right)$ radiative corrections can be summarized by a $K$ factor multiplying the lowest order rate. The $K$ factor can be defined as

$$
K \equiv \frac{\sigma_{N L O}}{\sigma_{L O}}
$$

From Fig. 4, it is clear that the $K$ factor computed in Ref. [3], where only the $\gamma$ exchange was considered, can be used at $\sqrt{s}=500 \mathrm{GeV}$. The $K$ factor depends only on the mass of the produced Higgs particle, and not on the SUSY parameters in the limit in which only the photon exchange contribution is important and so the results for the Standard Model Higgs boson can be used to obtain the $\mathcal{O}\left(\alpha_{s}\right)$ corrections. The only dependence on the renormalization scale $\mu$ is in the strong coupling constant, $\alpha_{s}(\mu)$. We will take $\mu=M_{t}=175 \mathrm{GeV}$ everywhere. A useful numerical fit to the $K$ factor of Ref. [3], valid for $\sqrt{s}=500 \mathrm{GeV}$, is, 


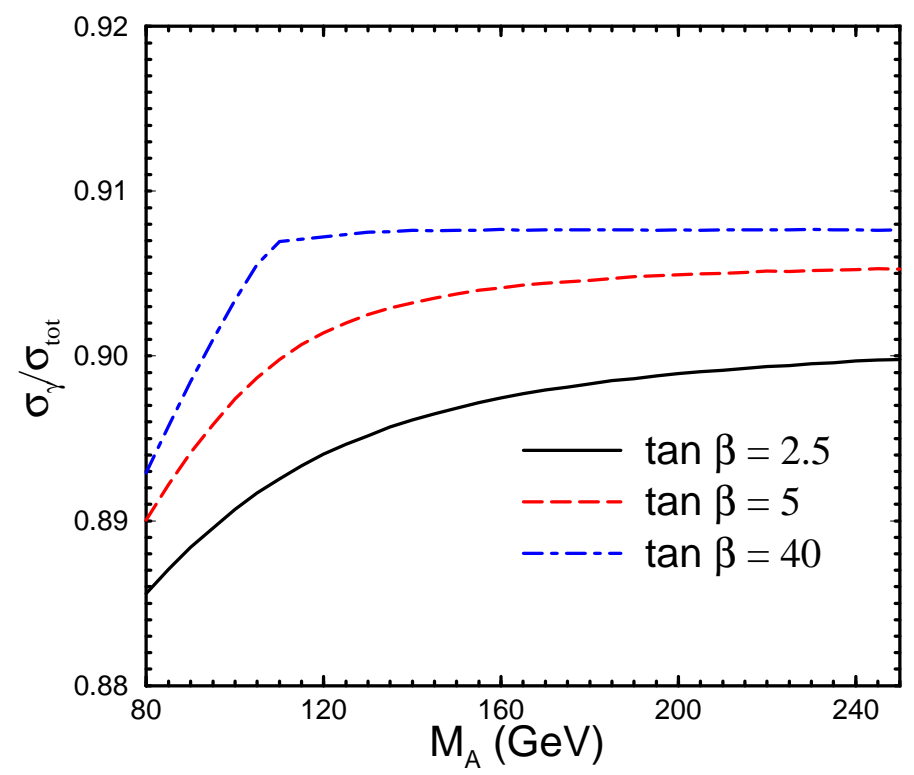

Figure 4: Dominance of the photon contribution to the process $e^{+} e^{-} \rightarrow t \bar{t} h^{0}$ at $\sqrt{s}=500 \mathrm{GeV}$. The squarks are assumed to have a common mass, $M_{S}=500 \mathrm{GeV}$ and the scalar mixing parameters are set to zero.

$$
\left.K\right|_{\sqrt{s}=500 \mathrm{GeV}^{\sim}} 1+\alpha_{s}\left(\mu^{2}\right)\left\{\frac{64}{9} \frac{M_{t}}{\sqrt{\left(\sqrt{s}-M_{h}\right)^{2}-4 M_{t}^{2}}}+a_{0}+a_{1} r+a_{2} r^{2}\right\}
$$

where

$$
r \equiv \frac{M_{h}}{100 \mathrm{GeV}}
$$

and

$$
\begin{aligned}
& a_{0}=-1.760, \\
& a_{1}=-.004, \\
& a_{2}=-.133 .
\end{aligned}
$$

This fit (valid only at $\sqrt{s}=500 \mathrm{GeV}$ ), is an excellent approximation to the exact $K$ factor of Refs. [3, 14. Fig. 5 shows that the QCD corrections increase the rate significantly at $\sqrt{s}=$ $500 \mathrm{GeV}$. At $\sqrt{s} \sim 500 \mathrm{GeV}$, much (but not all) of the $K$ factor can be accounted for by the threshold contribution to $t \bar{t}$ production. The first term in Eq. (12) is the threshold contribution to the $K$ factor [ [⿴囗 , which is plotted as the curve $K_{\text {threshold }}$ in Fig. 5, and the rising of the $K$ 


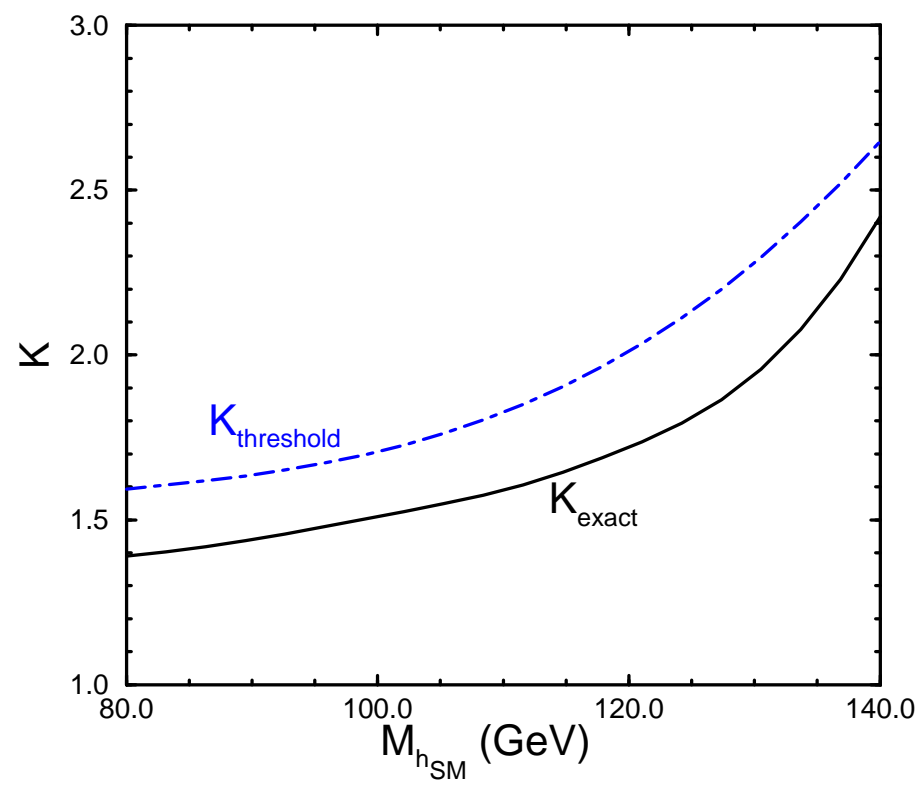

Figure 5: $K$ factor for the process $e^{+} e^{-} \rightarrow t \bar{t} h_{S M}$ at $\sqrt{s}=500 \mathrm{GeV}$. This figure uses $\alpha_{s}\left(M_{t}\right)=$ 0.11164. $K_{\text {exact }}$ is the result of Ref. [3] and $K_{\text {threshold }}$ includes only the $t \bar{t}$ threshold contribution. 41]

factor signaling the Coulomb singularity as the threshold is approached, $\sqrt{s} \sim 2 M_{t}+M_{h_{S M}}$, is clearly seen.

We can now compute the rates for $e^{+} e^{-} \rightarrow t \bar{t} h_{i}^{0}$ production including the $\mathcal{O}\left(\alpha_{s}\right)$ corrections. In Figs. 6 and 7, we show the lowest order and the radiatively corrected rates. All contributions to the lowest order rate ( $Z$ exchange, $A^{0} h_{i}^{0}$ production, etc) are included and the next-to-leading order result is computed by multiplying the lowest order rate by the $K$ factor of Fig. 5. Since the dominant contribution is photon exchange, this should be an excellent approximation. The rate for $t \bar{t} h^{0}$ is enhanced at small $\tan \beta$, while the rate for $t \bar{t} H^{0}$ production is enhanced at large $\tan \beta$. The rate is never larger than a few $f b$, making this process extremely challenging to observe.

Although the processes $e^{+} e^{-} \rightarrow t \bar{t} h^{0}$ and $e^{+} e^{-} \rightarrow t \bar{t} H^{0}$ have small rates, the signatures are distinctive since the final state will be predominantly $W^{+} W^{-} b \bar{b} b \bar{b}$ and so may be observable with a small number of events. In Fig. 8, we show the region of the $M_{A}-\tan \beta$ plane where the rates for either process are larger than $0.75 \mathrm{fb}$ and we see that this includes much of the parameter space. We have everywhere set the mixing in the squark and Higgs sectors to zero. Our results are relatively insensitive to this assumption.

At $\sqrt{s}=1 \mathrm{TeV}$, the cross section is no longer dominated by photon exchange and the $Z$ 


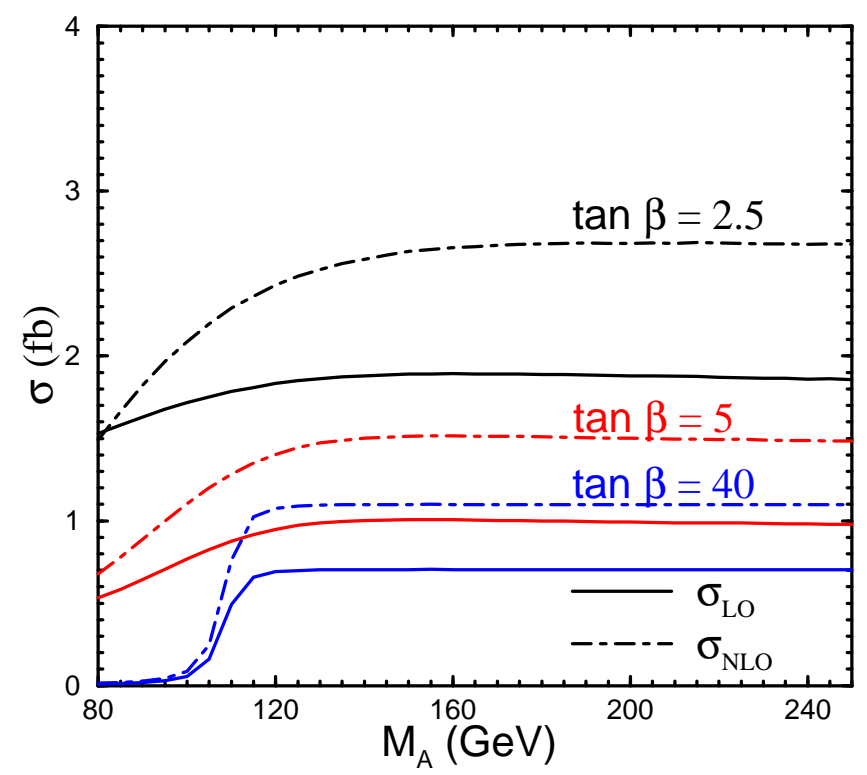

Figure 6: Cross section for $e^{+} e^{-} \rightarrow t \bar{t} h^{0}$ production at $\sqrt{s}=500 \mathrm{GeV}$ at lowest order and at NLO for different values of $\tan \beta$. We take $\alpha_{s}\left(M_{t}\right)=0.11164$ and use the $K$ factor of Ref. [3]. The squarks are taken to have a common mass, $M_{S}=500 \mathrm{GeV}$, and the mixing parameters are set to zero.

exchange becomes important. The ratio of the cross section computed using only the photon exchange to the total cross section varies between 70 and $80 \%$. However, the contribution from the $A^{0} h_{i}^{0}$ production of Fig. 3 is always less than $1 \%$. In Ref. 田, the $K$ factor for Standard Model Higgs production was computed including the effects of $Z$ exchange. Using $\alpha_{s}\left(M_{t}\right)=0.11164$, the result of Ref. [1] is that the $K$ factor at $\sqrt{s}=1 \mathrm{TeV}$ is roughly a constant, $K(1 \mathrm{TeV}) \sim 0.94$. We will use this result in our plots. Since the radiation of a Higgs boson from the $Z$ boson (the second diagram in Fig. 2) is small, the $K$ factor is again approximately independent of the SUSY parameters and depends only on the mass of the produced Higgs boson. At high energy where $\sqrt{s}$ is not close to the kinematic threshold, the dominant contribution to the QCD corrections is from the Higgs vertex correction computed in Ref. [12]. In this limit the $K$ factor is roughly,

$$
K \sim 1-\frac{3 \alpha_{s}(\mu)}{\pi} \sim 0.9
$$

in agreement with the result found from the complete calculation for $\sqrt{s}=1$ TeV. [3, 4] Figs. 9 and 10 show the rates for $e^{+} e^{-} \rightarrow t \bar{t} h_{i}^{0}$ at $\sqrt{s}=1 \mathrm{TeV}$. The cross section for either $e^{+} e^{-} \rightarrow t \bar{t} h^{0}$ or $e^{+} e^{-} \rightarrow t \bar{t} H^{0}$ is greater than $2 \mathrm{fb}$ throughout most of the $M_{A}-\tan \beta$ plane. For $M_{A}>120 \mathrm{GeV}$, 


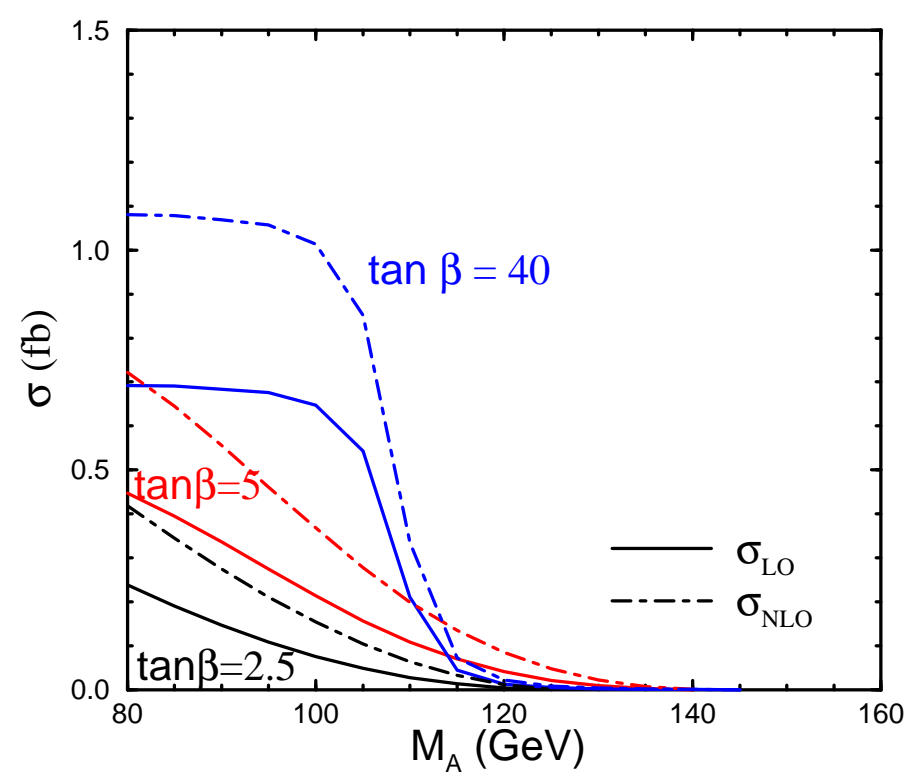

Figure 7: Cross section for $e^{+} e^{-} \rightarrow t \bar{t} H^{0}$ production at $\sqrt{s}=500 \mathrm{GeV}$ at lowest order and at NLO for different values of $\tan \beta$. We take $\alpha_{s}\left(M_{t}\right)=0.11164$ and use the $K$ factor of Ref. [3]. The squarks are taken to have a common mass, $M_{S}=500 \mathrm{GeV}$ and the mixing parameters are set to zero.

the rate for $e^{+} e^{-} \rightarrow t \bar{t} h^{0}$ is relatively insensitive to $\tan \beta$ and is always between 2.4 and $2.8 \mathrm{fb}$. The rate for $e^{+} e^{-} \rightarrow t \bar{t} H^{0}$ is always less than $1 \mathrm{fb}$ for $M_{A}>120 \mathrm{GeV}$. Projected luminosities for a $1 \mathrm{TeV}$ machine are around $200 \mathrm{fb}^{-1}$, which would give more than $400 t \bar{t} h^{0}$ events/year.

The dependence of the process $e^{+} e^{-} \rightarrow t \bar{t} h^{0}$ on the energy of the Higgs boson is shown in Fig. 11. As $\tan \beta$ becomes large, the distribution is peaked at larger values of $x_{h}=2 E_{h} / \sqrt{s}$. This is a kinematic effect due to the changing of the Higgs boson mass, since as $\tan \beta$ is increased, the Higgs mass increases with increasing $M_{A}$.

\section{$4 e^{+} e^{-} \rightarrow t \bar{t} A^{0}$}

The production of a pseudoscalar in association with a heavy quark pair proceeds through $\gamma$ and $Z$ exchange, and also through the subprocesses, $e^{+} e^{-} \rightarrow A^{0} h_{i}^{0} \rightarrow A^{0} Q \bar{Q}$, where $h_{i}^{0}=h^{0}, H^{0}$. The rate is given by, [2]

$$
\frac{d \sigma\left(e^{+} e^{-} \rightarrow Q \bar{Q} A^{0}\right)}{d x_{A}}=N_{c} \frac{\sigma_{0}}{(4 \pi)^{2}}\left\{\left[Q_{e}^{2} Q_{Q}^{2}+\frac{2 Q_{e} Q_{Q} v_{e} v_{Q}}{1-M_{Z}^{2} / s}+\frac{\left(v_{e}^{2}+a_{e}^{2}\right)\left(v_{Q}^{2}+a_{Q}^{2}\right)}{\left(1-M_{Z}^{2} / s\right)^{2}}\right] F_{1}\right.
$$




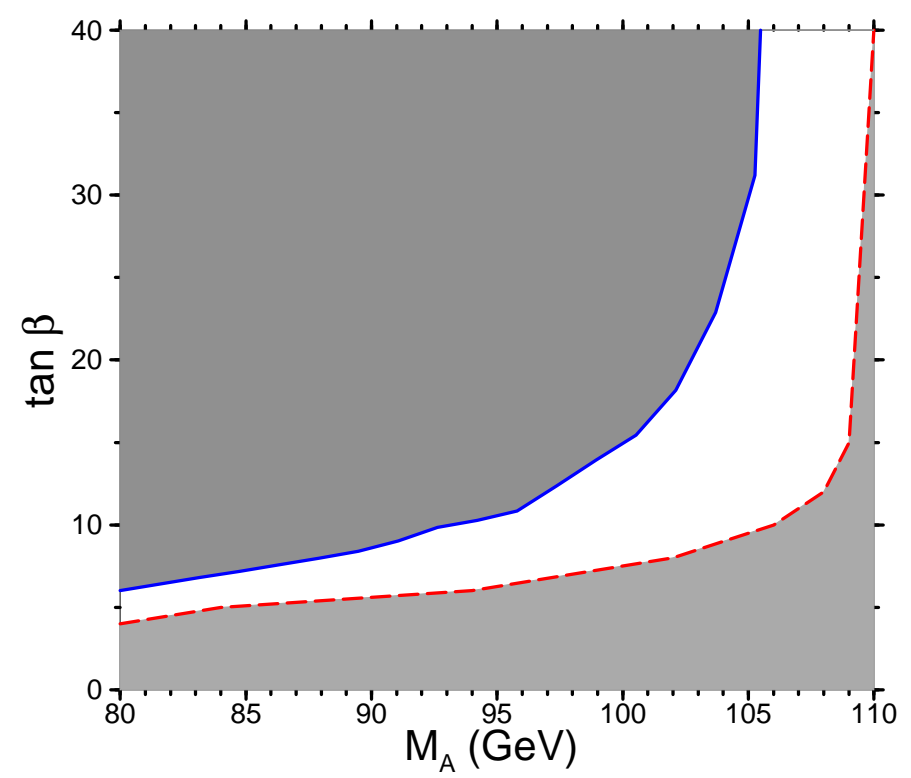

Figure 8: Regions in the $M_{A}-\tan \beta$ plane where the cross section for $e^{+} e^{-} \rightarrow t \bar{t} h_{i}^{0},\left(h_{i}^{0}=h^{0}, H^{0}\right)$, production is larger than $0.75 \mathrm{fb}$ at $\sqrt{s}=500 \mathrm{GeV}$. The upper left hand region results from $e^{+} e^{-} \rightarrow t \bar{t} H^{0}$, while the region at the lower right is the result from $e^{+} e^{-} \rightarrow t \bar{t} h^{0}$. All NLO QCD corrections are included. The squarks are taken to have a common mass, $M_{S}=500 \mathrm{GeV}$ and the mixing parameters are set to zero.

$$
\left.+\frac{v_{e}^{2}+a_{e}^{2}}{\left(1-M_{Z}^{2} / s\right)^{2}}\left(\frac{1}{16 c_{W}^{2} s_{W}^{2}}\right)\left[\left(2 I_{3 L}^{Q}\right)^{2} F_{2}+F_{3}+2 I_{3 L}^{Q} F_{4}\right]\right\},
$$

where $x_{A}=2 E_{A} / \sqrt{s}$. Expressions for the coefficients, $F_{i}\left(x_{A}\right)$, are given in Appendix B.

The cross section for $e^{+} e^{-} \rightarrow t \bar{t} A^{0}$ is less than $10^{-2} \mathrm{fb}$ at $\sqrt{s}=500 \mathrm{GeV}$ for all values of $\tan \beta$ and $M_{A}$ in the MSSM and so almost certainly will not be observable. However, the production of a scalar or of a pseudoscalar in association with a $t \bar{t}$ pair has been shown to be a useful mechanism for determining the CP coupling of the Higgs boson. 13] In Fig. 12, we show the normalized distribution of the Higgs boson energy for the lightest scalar of the MSSM and for the pseudoscalar of the MSSM. T The shape of the distributions is quite different, suggesting that this may be a useful method for discriminating between scalar and pseudoscalar Higgs- fermion couplings. For this to be practical, of course, requires a model where the pseudoscalar-fermion coupling is much larger than in the MSSM.

\footnotetext{
${ }^{5}$ These plots include contributions from the $e^{+} e^{-} \rightarrow h^{0} A^{0}$ resonance, which is specific to the MSSM.
} 


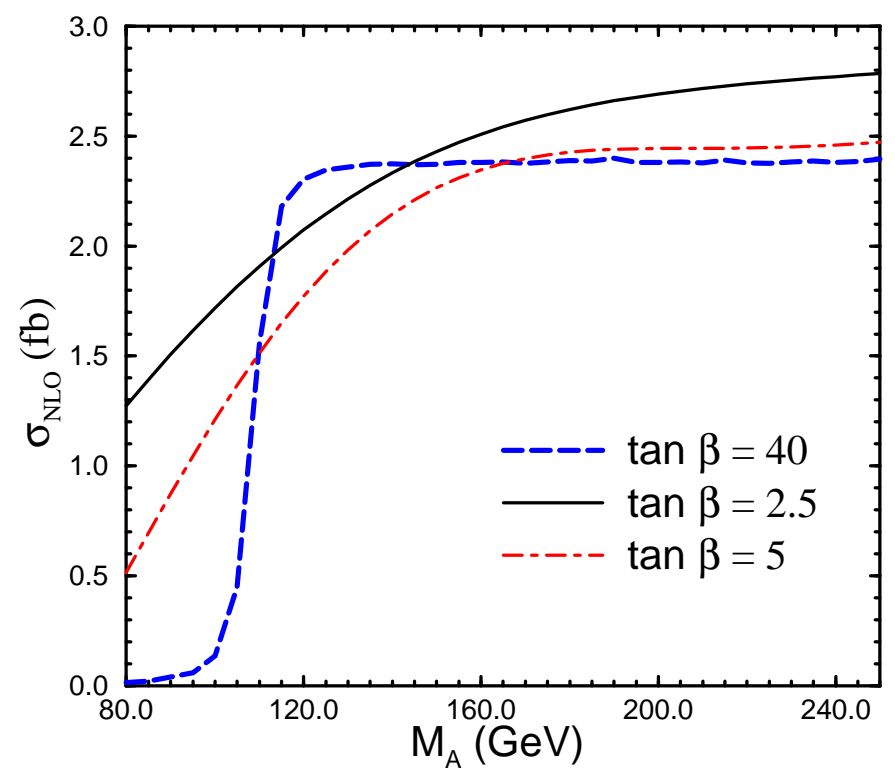

Figure 9: Next-to-leading order result for $e^{+} e^{-} \rightarrow t \bar{t} h^{0}$ at $\sqrt{s}=1 \mathrm{TeV}$ using $K=0.94$. The squarks are taken to have a common mass, $M_{S}=500 \mathrm{GeV}$.

\section{$5 \quad e^{+} e^{-} \rightarrow b \bar{b} h^{0}$ and $e^{+} e^{-} \rightarrow b \bar{b} H^{0}$}

In the MSSM, the Higgs boson can also be produced in association with $b \bar{b}$ pairs and the differential cross section is given by Eq. (9). The physics is significantly different from the production with a $t \bar{t}$ pair, however. In the case of the $b$ quark, the contribution of Fig. 3 receives a large resonant enhancement from the decay, $A^{0} \rightarrow b \bar{b}$. This enhancement occurs when $M_{h_{i}} \sim M_{A}$ and $x_{h} \sim 1$ and so is relevant for $M_{A}$ below about $120 \mathrm{GeV}$ for $e^{+} e^{-} \rightarrow b \bar{b} h^{0}$.

We can estimate the resonant contribution in the narrow width approximation,

$$
\left.\sigma\left(e^{+} e^{-} \rightarrow b \bar{b} h_{i}^{0}\right)\right|_{N W} \sim \frac{3 g_{b b h_{i}}^{2} g_{Z A h_{i}}^{2} \sigma_{0} \hat{\beta}}{128 \pi c_{W}^{2} s_{W}^{2}} \frac{v_{e}^{2}+a_{e}^{2}}{\left(1-M_{Z}^{2} / s\right)^{2}}\left(\frac{M_{A}}{\Gamma_{A}}\right)\left(3 \frac{M_{h_{i}}^{2}}{s}+\frac{M_{A}^{2}}{s}-1\right),
$$

where $\hat{\beta}$ is given by Eq.(22) in Appendix B and $\Gamma_{A}$ is the total decay width of the pseudoscalar $A^{0}$, shown in Fig. 13. For the parameters considered here, the pseudoscalar is a very narrow resonance. This process is sensitive to the couplings of the $Z$ to the $A^{0} h_{i}^{0}$ pair in the MSSM, ${ }^{6}$

$$
g_{Z A h}=\cos (\beta-\alpha),
$$

\footnotetext{
${ }^{6}$ The $g_{Z A h_{i}}$ couplings are normalized to the $Z Z h_{S M}$ coupling.
} 


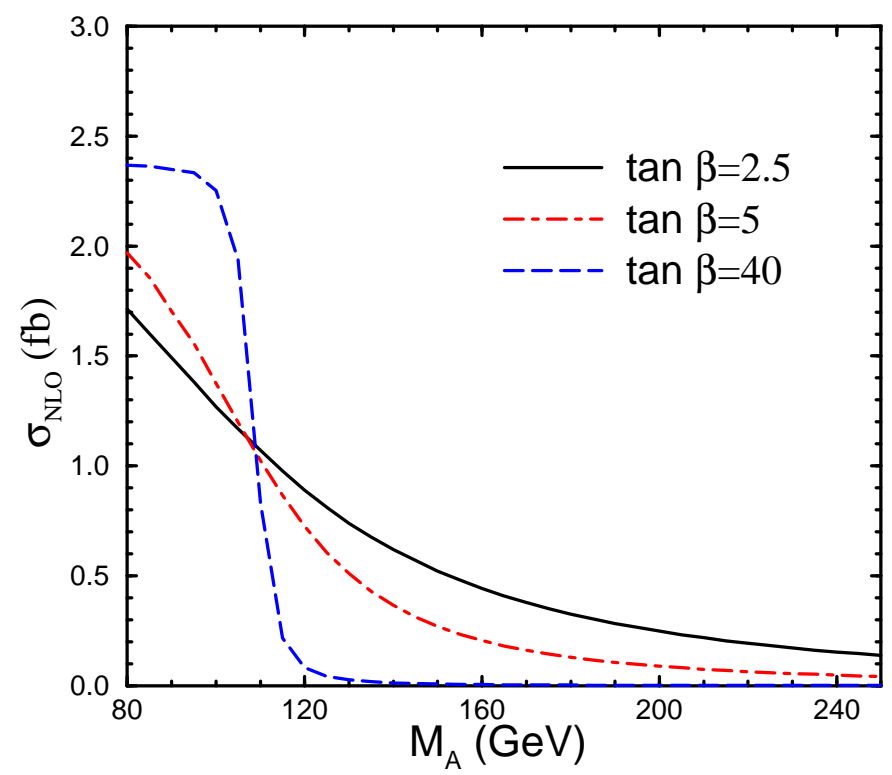

Figure 10: Next-to-leading order result for $e^{+} e^{-} \rightarrow t \bar{t} H^{0}$ at $\sqrt{s}=1 \mathrm{TeV}$ using $K=0.94$. The squarks are taken to have a common mass, $M_{S}=500 \mathrm{GeV}$.

$$
g_{Z A H}=-\sin (\beta-\alpha)
$$

along with the $b \bar{b} h_{i}^{0}$ Yukawa couplings.

Figs. 14 and 15 show the different contributions to the process $e^{+} e^{-} \rightarrow b \bar{b} h^{0}$ for a low and a high value of $\tan \beta$, at $\sqrt{s}=500 \mathrm{GeV}$ \. The curve labelled " $N W$ " is the narrow width approximation of Eq. (17), labelled as "total", while the curve labelled "AhZ" is only the contribution from the square of the diagram of Fig. 3. At small $M_{A}(<120 \mathrm{GeV})$, the narrow width approximation is an excellent approximation to the total rate for these values of $\tan \beta$. For smaller $\tan \beta$, the narrow width approximation becomes increasingly inaccurate, since the $Z$ exchange contribution becomes more and more relevant. Also at large $M_{A}$, the rate is given predominantly by the $Z$ boson exchange contribution and is typically between 5 and $10 \mathrm{fb}$. In Ref. [2], only the contribution from the Higgs coupling to the $b$ quark, (the first diagram of Fig. 2), was included in the figures. This is only valid for $M_{A}$ away from the resonance region. For $M_{A}$ near the resonance region, Ref. [2] significantly underestimates the size of this process.

\footnotetext{
${ }^{7}$ We do not consider values of $\tan \beta$ smaller than 5 since the values of $M_{h}$ corresponding to the resonance region of the $b b h^{0}$ production mode would be already experimentally excluded.

${ }^{8}$ In the Standard Model, the $Z$ resonance is the dominant contribution.
} 


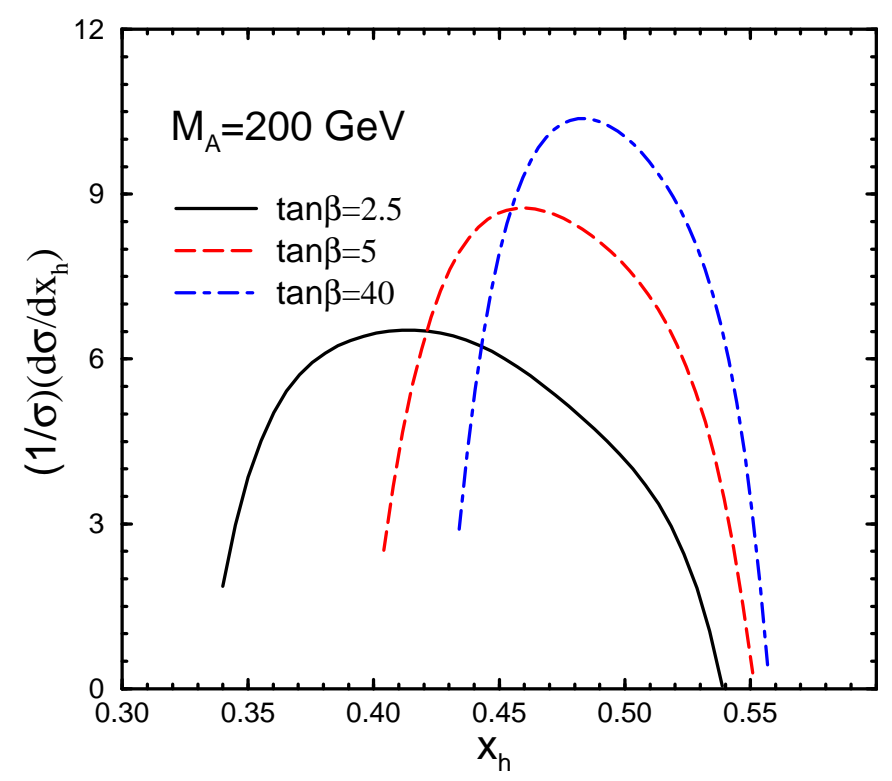

Figure 11: Dependence of the process $e^{+} e^{-} \rightarrow t \bar{t} h^{0}$ at $\sqrt{s}=500 \mathrm{GeV}$ on the energy of the Higgs boson, $x_{h}=2 E_{h} / \sqrt{s}$, for $M_{A}=200 \mathrm{GeV}$. The squarks are assumed to have a common mass, $M_{S}=500 \mathrm{GeV}$ and the scalar mixing parameters are set to zero.

In the narrow width approximation, the QCD corrections to the rate are trivially included by including the QCD corrections to the pseudo-scalar width and this is done in Figs. 14 and 15. The QCD $\mathcal{O}\left(\alpha_{s}\right)$ corrections to $\Gamma_{A}$ can be found in Ref. [6]. Away from the resonance, (large $M_{A}$ ), inclusion of the QCD corrections would require a complete calculation, which we do not include in the present analysis since the interesting region is near the resonance where the rate is enhanced.

For the heavy Higgs production, the narrow width approximation is an excellent approximation for all values of $\tan \beta$ so the QCD corrections can be accurately included everywhere. The rate for $b \bar{b} H^{0}$ production is shown in Fig. 16. For $\tan \beta<5$, the cross section is larger than $20 \mathrm{fb}$ even for $M_{A} \sim 200 \mathrm{GeV}$. For $\tan \beta>5$, the rate is greater than $20 \mathrm{fb}$ for $M_{A}>110 \mathrm{GeV}$. This process can potentially be used to probe the couplings of the heavier neutral Higgs boson and obtain a precise measurement of the product of the Higgs couplings, $g_{b b H} g_{Z A H}$.

The same type of behavior is still present if we vary over the possible values of the scalar masses and mixing parameters or if we look at $\sqrt{s}=1 \mathrm{TeV}$. 


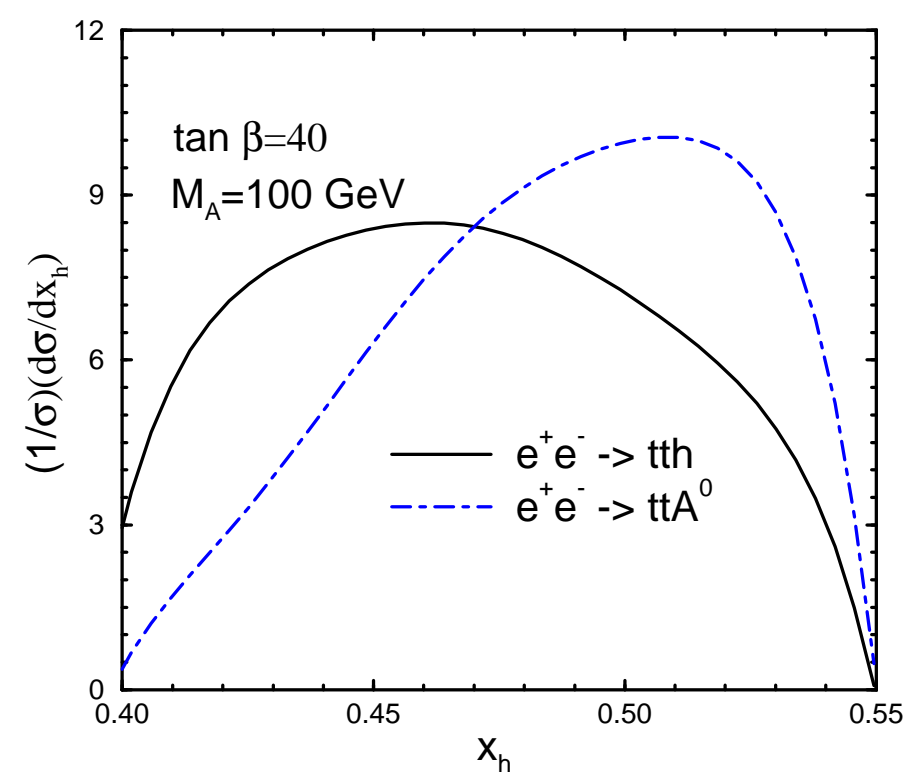

Figure 12: Distribution in scalar energy for the pseudoscalar, $A^{0}$ and the lightest neutral scalar, $h^{0}$ of the MSSM at $\sqrt{s}=500 \mathrm{GeV}$. The squarks are taken to have a common mass, $M_{S}=500 \mathrm{GeV}$.

\section{$6 \quad e^{+} e^{-} \rightarrow b \bar{b} A^{0}$}

We can safely work in the narrow width approximation also for the case of $e^{+} e^{-} \rightarrow b \bar{b} A^{0}$ production. In fact, in this case the $h_{i}^{0}$ resonances are completely dominant and the exact cross section can be distinguished from the one obtained using the narrow width approximation only at very high values of $\tan \beta$. The cases of $\tan \beta=5$ and $\tan \beta=40$ are illustrated in Figs. 17 and 18. We can see that for $\tan \beta=5$ the exact cross section is indistinguishable from the one obtained in the narrow width approximation and the only relevant contribution is given by $e^{+} e^{-} \rightarrow A^{0} h_{i}^{0} \rightarrow A b \bar{b}$.

Unlike $t \bar{t} A^{0}$ production, the process $e^{+} e^{-} \rightarrow b \bar{b} A^{0}$ is not suppressed relative to $e^{+} e^{-} \rightarrow$ $b \bar{b} h^{0}$ production. The resonant behavior of the $b b A^{0}$ production mode is very stable with respect to the variation of the scalar mass and mixing parameters of the MMSM. Different parametrizations only affect the values of $M_{h}$ and $M_{H}$ at resonance. 


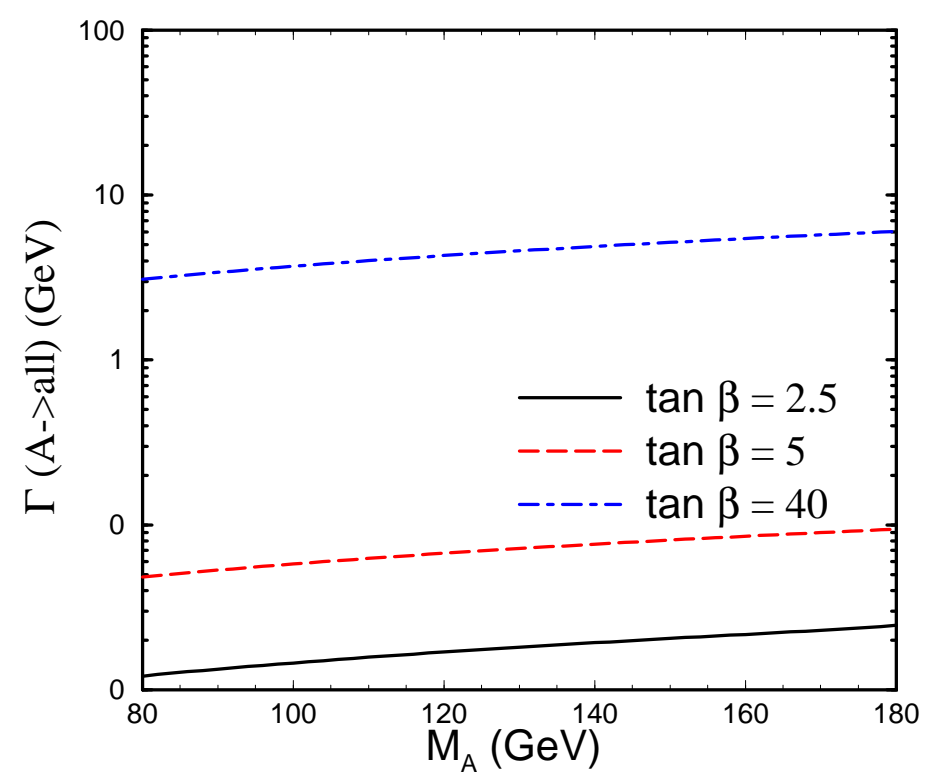

Figure 13: Total width of the pseudoscalar, $A^{0}$, in the MSSM including NLO QCD corrections and assuming that there are no open channels for the $A^{0}$ to decay into SUSY particles. The squarks are assumed to have a common mass, $M_{S}=500 \mathrm{GeV}$ and the scalar mixing parameters are set to zero.

\section{Conclusion}

We have considered the production of the Higgs bosons of the MSSM in association with a heavy quark pair. The processes $e^{+} e^{-} \rightarrow t \bar{t} h^{0}\left(H^{0}\right)$ have rates around $1 \mathrm{fb}$ at $\sqrt{s}=500 \mathrm{GeV}$ over much of the parameter space of the MSSM. They can reach rates of $2 \mathrm{fb}$ in selected regions of the parameter space at $\sqrt{s}=500 \mathrm{GeV}$ and over most of the parameter space at $\sqrt{s}=1 \mathrm{TeV}$. Observation of these processes could provide a precise measurement of the $t t h_{i}^{0}$ couplings in a region not accessible at LEP2 and with $50 \mathrm{fb}^{-1}$, the $t t h^{0}$ process could give a $90 \%$ c.l. measurement with a statistical error of

$$
\frac{\delta g_{t t h}}{g_{t t h}}< \pm 10 \%
$$

over much of the $M_{A}-\tan \beta$ parameter space. The shape of the Higgs boson energy spectrum in these processes is quite sensitive to the value of $\tan \beta$. The rate for $e^{+} e^{-} \rightarrow t \bar{t} A^{0}$ is too small to be observed.

For $M_{A}>120 \mathrm{GeV}$, the rate for $e^{+} e^{-} \rightarrow b \bar{b} h^{0}$ is also small, on the order of a few $f b$ at $\sqrt{s}=500 \mathrm{GeV}$. However, in the resonance region, $M_{A} \sim M_{h}, b \bar{b} h^{0}$ production receives a 


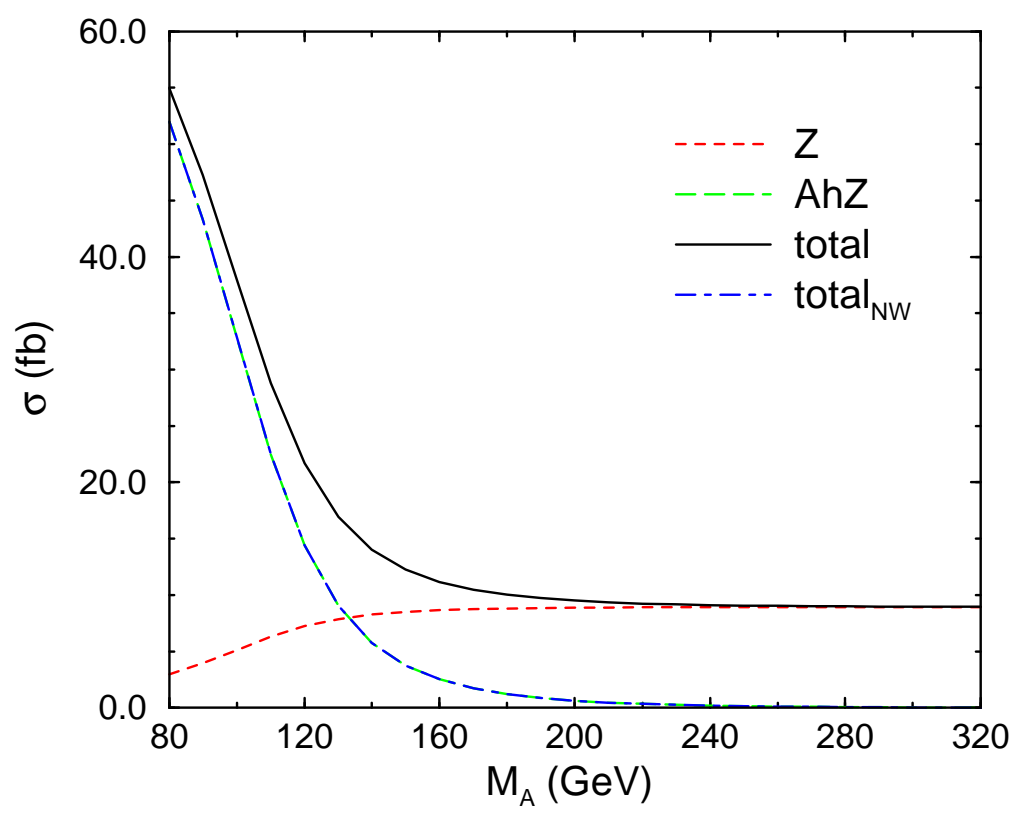

Figure 14: Contributions to $e^{+} e^{-} \rightarrow b \bar{b} h^{0}$ at $\sqrt{s}=500 \mathrm{GeV}$, for $\tan \beta=5$. The curve labelled 'NW' is the narrow width approximation of Eq. (17) and it includes QCD corrections in the resonance region. The squarks are assumed to have a common mass, $M_{S}=500 \mathrm{GeV}$ and the scalar mixing parameters are set to zero.

large enhancement. This leads to the possibility of probing above the threshold accessed by LEP2. The rates for $e^{+} e^{-} \rightarrow b \bar{b} H^{0}$ and $e^{+} e^{-} \rightarrow b \bar{b} A^{0}$ are greater than $20 \mathrm{fb}$ throughout much of the MSSM parameter space and offer a unique window for studying the couplings of the pseudoscalar and heavier neutral Higgs bosons. 


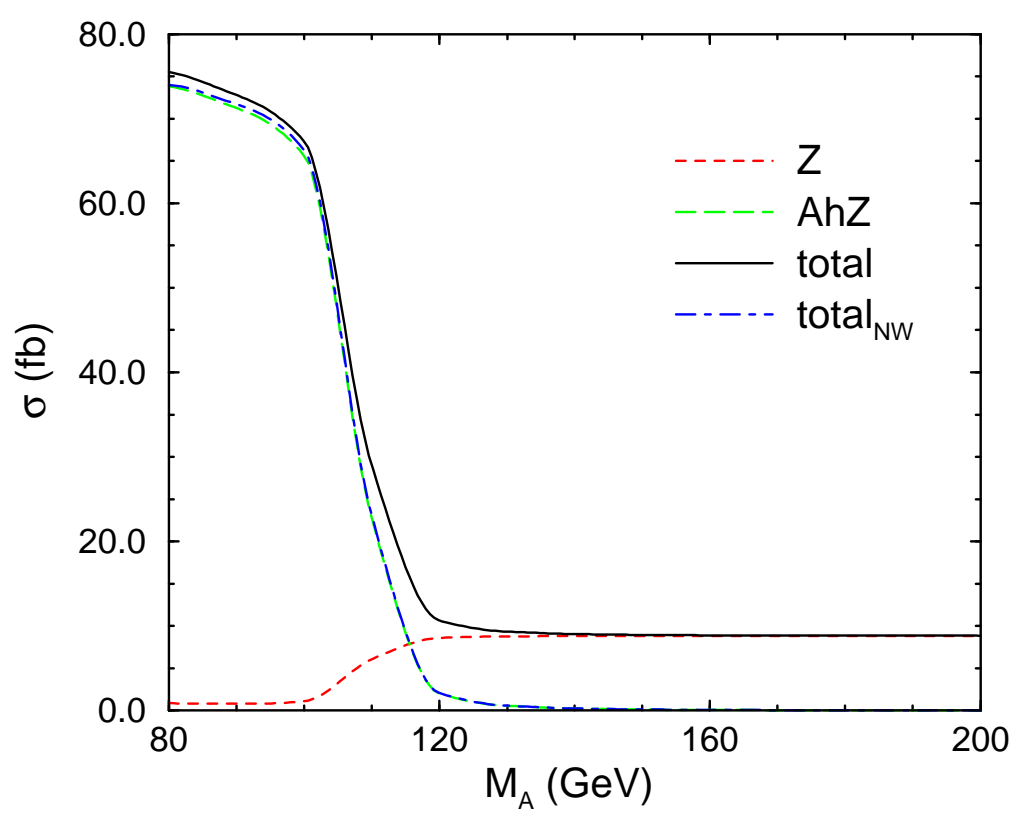

Figure 15: Contributions to $e^{+} e^{-} \rightarrow b \bar{b} h^{0}$ at $\sqrt{s}=500 \mathrm{GeV}$, for $\tan \beta=40$. The curve labelled 'NW' is the narrow width approximation of Eq. (17) and it includes QCD corrections in the resonance region. The squarks are assumed to have a common mass, $M_{S}=500 \mathrm{GeV}$ and the scalar mixing parameters are set to zero.

\section{A Coefficients for $e^{+} e^{-} \rightarrow Q \bar{Q} h_{i}^{0}$ Production}

The cross section for the process $e^{+} e^{-} \rightarrow Q \bar{Q} h_{i}^{0}\left(h_{i}^{0}=h^{0}, H^{0}\right)$ is given in Eq. (9) in terms of coefficients, $G_{i}$. The coefficients $G_{1}$ and $G_{2}$ describe the radiation of the Higgs boson from the heavy quark (both photon and $Z$ boson exchange) and are given by, [2, 迎]

$$
\begin{aligned}
& G_{1}=\frac{2 g_{Q Q h_{i}}^{2}}{s^{2}\left(\hat{\beta}^{2}-x_{h}^{2}\right) x_{h}}\left(-4 \hat{\beta}\left(4 M_{Q}^{2}-M_{h_{i}}^{2}\right)\left(2 M_{Q}^{2}+s\right) x_{h}+\right. \\
& \left.\quad\left(\hat{\beta}^{2}-x_{h}^{2}\right)\left(16 M_{Q}^{4}+2 M_{h_{i}}^{4}-2 M_{h_{i}}^{2} s x_{h}+s^{2} x_{h}^{2}-4 M_{Q}^{2}\left(3 M_{h_{i}}^{2}-2 s-2 s x_{h}\right)\right) \Lambda\right), \\
& G_{2}=\frac{-2 g_{Q Q h_{i}}^{2}}{s^{2}\left(\hat{\beta}^{2}-x_{h}^{2}\right) x_{h}}\left(\hat{\beta} x_{h}\left(-96 M_{Q}^{4}+24 M_{Q}^{2} M_{h_{i}}^{2}-\left(-M_{h_{i}}^{2}+s+s x_{h}\right)\left(-\hat{\beta}^{2}+x_{h}^{2}\right)\right)+\right. \\
& \left.2\left(\hat{\beta}^{2}-x_{h}^{2}\right)\left(24 M_{Q}^{4}+2\left(M_{h_{i}}^{4}-M_{h_{i}}^{2} s x_{h}\right)+M_{Q}^{2}\left(-14 M_{h_{i}}^{2}+12 s x_{h}+s x_{h}^{2}\right)\right) \Lambda\right),
\end{aligned}
$$

where we have defined

\footnotetext{
${ }^{9} G_{1}, \ldots G_{6}$ agree with those of Ref. [2]. $G_{7}$ corrects a sign error in Ref. [2]. We thank J. Kalinowski for a discussion of Ref. [2].
} 


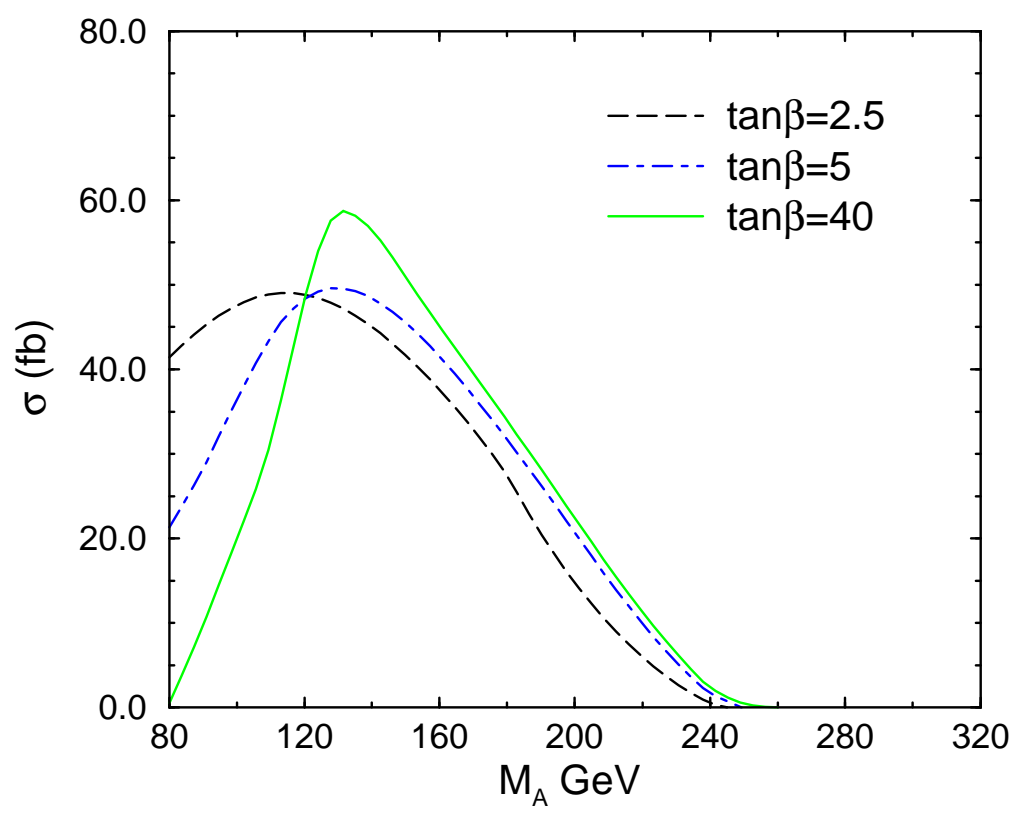

Figure 16: Cross section for $e^{+} e^{-} \rightarrow b \bar{b} H^{0}$ at $\sqrt{s}=500 \mathrm{GeV}$, including QCD corrections in the narrow width approximation. The squarks are assumed to have a common mass, $M_{S}=500 \mathrm{GeV}$ and the scalar mixing parameters are set to zero.

$$
\begin{aligned}
\Lambda & \equiv \log \left(\frac{x_{h}+\hat{\beta}}{x_{h}-\hat{\beta}}\right) \\
X_{i} & \equiv\left(M_{h_{i}}^{2}-M_{Z}^{2}+s-s x_{h}\right)
\end{aligned}
$$

and

$$
\hat{\beta}=\left\{\frac{\left[x_{h}^{2}-\left(x_{h}^{\min }\right)^{2}\right]\left[x_{h}^{\max }-x_{h}\right]}{x_{h}^{\max }-x_{h}+\frac{4 M_{Q}^{2}}{s}}\right\}^{1 / 2},
$$

with $x_{h}^{\min }=2 M_{h_{i}} / \sqrt{s}$ and $x_{h}^{\max }=1-4 M_{Q}^{2} / s+M_{h_{i}}^{2} / s$.

The other four coefficients, $G_{3}, \ldots, G_{6}$ describe the emission of a Higgs boson from the $Z$-boson (plus relative interference terms) and can be written in the following form,

$$
\begin{aligned}
G_{3}= & \frac{-2 \hat{\beta} g_{Z}^{2} M_{Q}^{2}}{M_{Z}^{2} s^{2} X_{i}^{2}}\left(4 M_{h_{i}}^{4}+12 M_{Z}^{4}+2 M_{Z}^{2} s x_{h}^{2}+s^{2}\left(-1+x_{h}\right) x_{h}^{2}-\right. \\
& \left.M_{h_{i}}^{2}\left(8 M_{Z}^{2}+s\left(-4+4 x_{h}+x_{h}^{2}\right)\right)\right),
\end{aligned}
$$




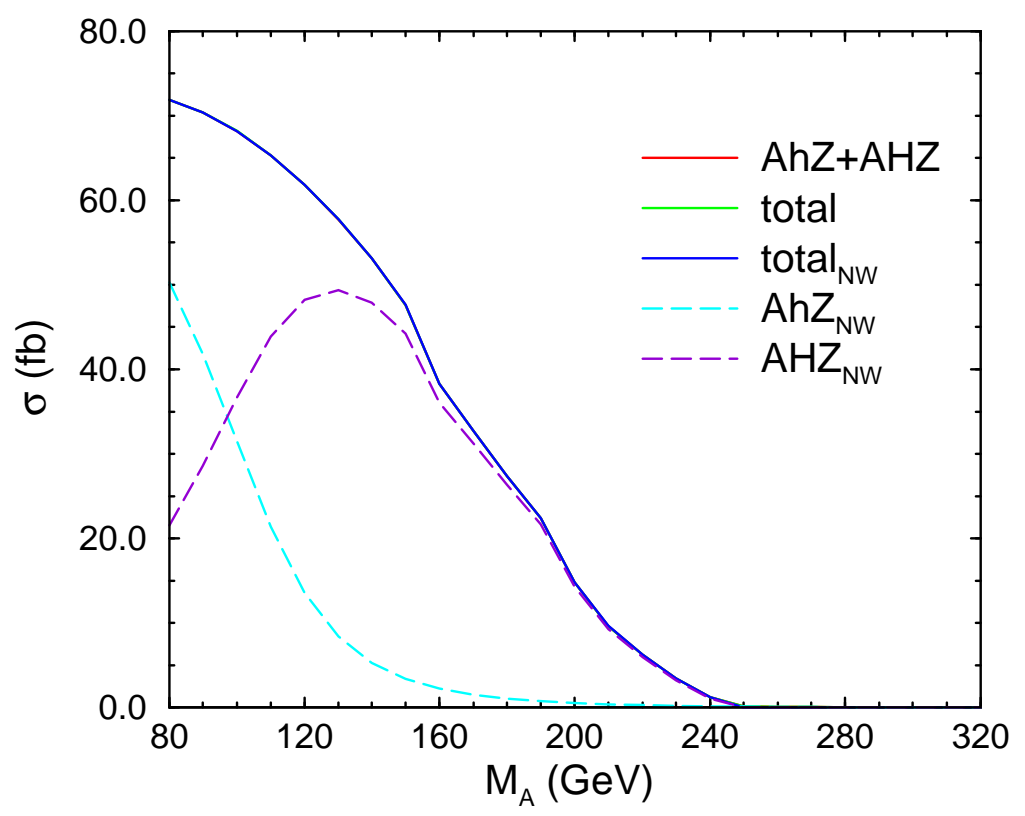

Figure 17: Total and partial contributions to the cross section for $e^{+} e^{-} \rightarrow b \bar{b} A$ at $\sqrt{s}=500 \mathrm{GeV}$ and $\tan \beta=5$, including QCD corrections. The squarks are assumed to have a common mass, $M_{S}=500 \mathrm{GeV}$ and the scalar mixing parameters are set to zero.

$$
\begin{aligned}
G_{4}= & \frac{\hat{\beta} g_{Z}^{2} M_{Z}^{2}}{6 s^{2} X_{i}^{2}}\left(48 M_{Q}^{2}+12 M_{h_{i}}^{2}-s\left(-24+\hat{\beta}^{2}+24 x_{h}-3 x_{h}^{2}\right)\right), \\
G_{5}= & \frac{4 g_{Q Q h_{i}} g_{Z} M_{Q}}{M_{Z} s^{2} X_{i}}\left(\hat{\beta} s\left(6 M_{Z}^{2}+x_{h}\left(-M_{h_{i}}^{2}-s+s x_{h}\right)\right)+\right. \\
& \left.2\left(M_{h_{i}}^{2}\left(M_{h_{i}}^{2}-3 M_{Z}^{2}+s-s x_{h}\right)+M_{Q}^{2}\left(-4 M_{h_{i}}^{2}+12 M_{Z}^{2}+s x_{h}^{2}\right)\right) \Lambda\right), \\
G_{6}= & \frac{-8 g_{Q Q h_{i}} g_{Z} M_{Q} M_{Z}}{s^{2} X_{i}}\left(\hat{\beta} s+\left(4 M_{Q}^{2}-M_{h_{i}}^{2}+2 s-s x_{h}\right) \Lambda\right),
\end{aligned}
$$

where $g_{Z}$ denotes the coupling of the Higgs boson to the $Z$ boson,

$$
g_{Z} \equiv\left(\sqrt{2} G_{F}\right)^{1 / 2} M_{Z} g_{Z Z h_{i}}
$$

where

$$
\begin{aligned}
g_{Z Z h} & =\sin (\beta-\alpha), \\
g_{Z Z H} & =\cos (\beta-\alpha) .
\end{aligned}
$$

The contribution from $A^{0} h_{i}^{0}$ production (see Fig. 3) is given by 


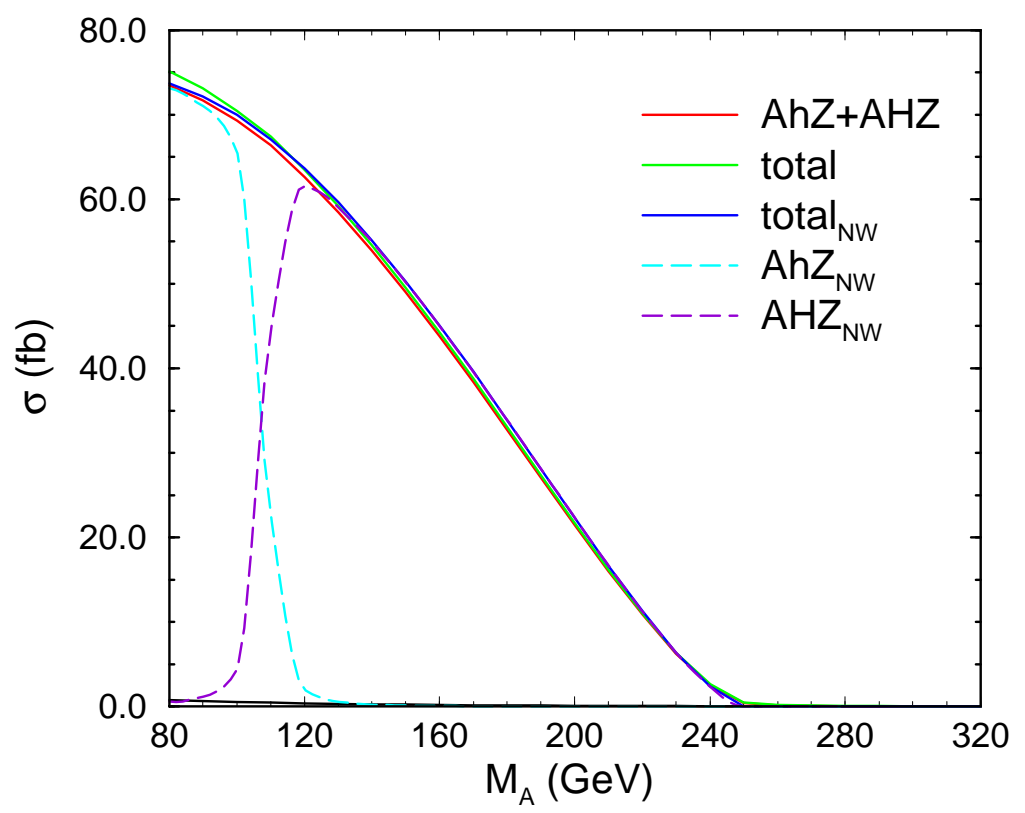

Figure 18: Total and partial contributions to the cross section for $e^{+} e^{-} \rightarrow b \bar{b} A$ at $\sqrt{s}=500 \mathrm{GeV}$ at $\tan \beta=40$, including QCD corrections. The squarks are assumed to have a common mass, $M_{S}=500 \mathrm{GeV}$ and the scalar mixing parameters are set to zero.

$$
\begin{aligned}
G_{7}= & -\frac{g_{Q Q A} g_{Z A h_{i}}}{Y_{i}}\left\{2 \beta\left(4 M_{h_{i}}^{2}-s x_{h}\right)\left[-g_{Z A h_{i}} g_{Q Q A} \frac{s x_{h}-s-M_{h_{i}}^{2}}{Y_{i}}-\frac{4 M_{Q} I_{3 L}^{Q} g_{Z Z h_{i}}}{M_{Z}}\right]\right. \\
& +\frac{4 I_{3 L}^{Q} g_{Q Q h_{i}}}{s}\left[2 s x_{h} \beta\left(s x_{h}-s-M_{h_{i}}^{2}\right)-4\left(M_{h_{i}}^{2}\left(s x_{h}-s-M_{h_{i}}^{2}\right)\right.\right. \\
& \left.\left.\left.+M_{Q}^{2}\left(4 M_{h_{i}}^{2}-s x_{h}^{2}\right)\right) \Lambda\right]\right\},
\end{aligned}
$$

where we have defined

$$
Y_{i}=M_{h_{i}}^{2}-M_{A}^{2}+s-s x_{h},
$$

while $g_{Q Q A}$ and $g_{Z A h_{i}}$ are given in Eqs. (7) (18).

\section{B Coefficients for $e^{+} e^{-} \rightarrow Q \bar{Q} A^{0}$}

The coefficients of Eq. (16) aretr

\footnotetext{
${ }^{10}$ Our result for $F_{4}\left(F_{3}\right)$ is a factor of $2(4)$ larger than the result of Ref. [2].
} 


$$
\begin{aligned}
F_{1} & =2 g_{Q Q A}^{2}\left\{\left[x_{A}-\frac{2 M_{A}^{2}}{s^{2} x_{A}}\left(s x_{A}-M_{A}^{2}+2 M_{Q}^{2}\right)\right] \Lambda+4 \frac{M_{A}^{2}}{s^{2}}\left(s+2 M_{Q}^{2}\right) \frac{\hat{\beta}}{\hat{\beta}^{2}-x_{A}^{2}}\right\}, \\
F_{2} & =2 g_{Q Q A}\left\{\hat{\beta}\left(1+x_{A}\right)-\frac{2}{s^{2} x_{A}}\left[2 M_{A}^{4}+M_{Q}^{2} s x_{A}^{2}-2 M_{A}^{2}\left(M_{Q}^{2}+s x_{A}\right)\right] \Lambda\right. \\
& \left.+\frac{M_{A}^{2}}{s^{2}} \frac{\hat{\beta}}{\hat{\beta}^{2}-x_{A}^{2}}\left(-24 M_{Q}^{2}+\hat{\beta}^{2} s-s x_{A}^{2}\right)\right\}, \\
F_{3} & =2 \hat{\beta}\left[\sum_{i} \frac{g_{Q Q h_{i}} g_{Z A h_{i}}}{Z_{i}}\right]^{2}\left(s x_{A}-s-M_{A}^{2}+4 M_{Q}^{2}\right)\left(4 M_{A}^{2}-s x_{A}^{2}\right), \\
F_{4} & =\frac{4}{s} \sum_{i}\left[\frac{g_{Q Q A} g_{Q Q h_{i}} g_{Z A h_{i}}}{Z_{i}}\right]\left\{\hat{\beta} s x_{A}\left(M_{A}^{2}+s-s x_{A}\right)\right. \\
& \left.-2\left[M_{A}^{4}+M_{Q}^{2} s x_{A}^{2}+M_{A}^{2}\left(-4 M_{Q}^{2}+s-s x_{A}\right)\right] \Lambda\right\},
\end{aligned}
$$

where we define

$$
Z_{i}=M_{A}^{2}-M_{h_{i}}^{2}+s-s x_{A}
$$

\section{References}

[1] K. Gaemers and G. Gounaris, Phys. Lett. B77 (1978) 379.

[2] A. Djouadi, J. Kalinowski and P. Zerwas, Zeit. Phys. C54 (1992) 255.

[3] S. Dawson and L. Reina, MADPH-98/1073, hep-ph/9808443.

[4] S. Dittmaier, M. Kramer, Y. Liao, M. Spira, and P. Zerwas, CERN-TH/98-277, hepph/9808433.

[5] J. Gunion, H. Haber, G. Kane, and S. Dawson, The Higgs Hunter's Guide, (AddisonWesley, Menlo Park, 1990.

[6] M. Spira, Fortsch. Phys. 46 (1998) 203.

[7] M. Carena and P. Zerwas, ed., CERN Yellow report, CERN-96-01, hep-ph/9602250.

[8] M. Carena, M. Quiros, and C. Wagner, Nucl. Phys. B461 (1996) 407; Y. Okada, M. Yamaguchi, and T. Yanagida, Prog. Theor. Phys. - bf 85 (1991); H. Haber and R. Hempfling, Phys. Rev. Lett. 66 (1991) 1815; J. Ellis, G. Ridolfi, and F. Zwirner, Phys. Lett. B257 (1991) 83; M. Carena, J. Espinosa, M. Quiros, anc C. Wagner, Phys. Lett. B355 (1995) 209. 
[9] A. Djouadi, J. Kalinowski, and M. Spira, Comp. Phys. Commun. 108 (1998) 56.

[10] D. Karlen, contribution to International Conference on High Energy Physics, July, 1998, Vancouver, Canada, http://ichep98.triumf.ca/private/convenors/transparancies/ plenary3_new.pdf .

[11] J. Erler and D. Pierce, Nucl. Phys. B526 (1998) 53 and D. Pierce, private communication.

[12] S. Dawson and L. Reina, Phys. Rev. D57 (1998) 5851.

[13] S. Bar-Shalom, D. Atwood, G. Eilam, R. Mendel, and A. Soni, Phys. Rev. D53 (1996) 1162; J. Gunion and X.-G. He, proceedings of 1996 DPF/DPB Summer Study on New Directions in High Energy Physics, Snowmass, Colorado, June 25-July 12, 1996. 